\section{Cantinas saludables para promover hábitos alimentarios en las escuelas}

Marcela Martinelli

Nora Aimaretti

Docentes investigadoras

de la Facultad de Bioquímica

y Ciencias Biológicas.

Universidad Nacional del Litoral.

Juan Villafañe

Presidente de la Federación

de Cooperadores Escolares del

Departamento La Capital, Santa Fe.
Universidad y salud /

Intervenciones

\section{Resumen}

La alimentación y la nutrición son muy importantes para promover y mantener la buena salud a lo largo de toda la vida. Las autoridades nacionales, provinciales y municipales reconocen la importancia de la Educación Alimentaria Nutricional (EAN) y la implementación de quioscos saludables en las escuelas. Sin embargo, hasta el momento muy poco se ha logrado en la provincia de Santa Fe. Desde 2008 existe un proyecto de ley que no logra sancionarse. La Ley 13295 sancionada en 2012 creó el Programa Provincial Integral de Prevención, Control y Asistencia de los Trastornos Alimentarios, pero aún no está reglamentada y es desconocida en los establecimientos escolares.

Un proyecto de extensión llevado adelante por la Universidad Nacional del Litoral (UNL) pretendió dar inicio a diferentes acciones que deben llevarse a cabo para mejorar la alimentación en la edad escolar y establecer buenos hábitos alimentarios que perduren a lo largo de la vida y permitan evitar enfermedades a futuro.

\author{
Palabras clave \\ - alimentación \\ - desayuno \\ - quioscos escolares \\ - educación alimentaria
}

\section{Introducción}

La alimentación y la nutrición son muy importantes para promover y mantener la buena salud a lo largo de toda la vida. Al mismo tiempo que millones de personas mueren de hambre en el mundo, la obesidad adquiere, según la Organización Mundial de la Salud (OMS) caracteres de epidemia (2003). En la actualidad, el sobrepeso y la obesidad son considerados uno de los problemas más importantes de salud pública a nivel mundial (OMS, 2006). Durante mucho tiempo, en Argentina, el sobrepeso y la obesidad fueron subestimados por considerarlos poco relevantes frente a los problemas de desnutrición existentes. Sin embargo, tal como ocurre en otras regiones de América Latina y a nivel mundial, el exceso de peso es actualmente un problema creciente en niños, adolescentes y adultos. Si bien se reconoce que en la etiología de la obesidad existen factores genéticos, metabólicos y ambientales, su elevada prevalencia ha sido asociada a la escasa actividad física y a hábitos alimentarios inapropiados. En lo que respecta a la alimentación, Argentina sigue siendo un país en donde hay abundancia de alimentos pero muchas personas no tienen acceso a cantidades suficientes y variadas de alimentos. Por su parte, otras personas pueden tener acceso a suficientes alimentos y sin embargo carecer de los conocimientos necesarios para alimentarse en forma apropiada, contribuyendo al cuidado de su salud y por lo tanto a mejorar la calidad de vida.

En el año 2007, el Ministerio de Salud de la Nación formuló el Plan Argentina Saludable, que contiene estrategias orientadas a la prevención de los principales factores de riesgo de las enfermedades crónicas no transmisibles, en particular el consumo de tabaco, la inactividad física y la alimentación inadecuada. En

cuanto a la alimentación, se observa que estos esfuerzos aún no son suficientes, tal como lo demuestra la Segunda Encuesta Nacional de Factores de Riesgo de 2009, que indica que más de la mitad de la población adulta $(53,4 \%)$ presenta exceso de peso, y esta prevalencia se ha incrementado con respecto al año 2005. Pero lo que se torna alarmante es la incidencia que tienen el sobrepeso y la obesidad en la población infantil. No existen actualmente datos oficiales y representativos a nivel nacional de lo que ocurre en niños en edad escolar; ${ }^{1}$ sin embargo, en diferentes estudios realizados en escolares de localidades de la provincia de Buenos Aires se ha encontrado una prevalencia de sobrepeso que oscila entre 21 y 34 \% y de obesidad entre 12 a 17 \% (Szer, 2010 y Kovalskys, 2013).

En este proyecto, que se realizó en dos escuelas primarias públicas de la ciudad de Santa Fe, se ha detectado una situación igualmente preocupante: $24,5 \%$ de los alumnos presentó sobrepeso y $21,5 \%$ obesidad. Esta elevada prevalencia de sobrepeso y obesidad se atribuye a que los niños argentinos hacen hoy menos actividad física, juegan menos al aire libre y han modificado sus hábitos alimentarios. Diversas investigaciones han demostrado que

1) Las últimas encuestas nacionales que aportan datos sobre el estado nutricional de la población argentina son la Encuesta Nacional de Nutrición y Salud 2005 (la población estudiada incluyó niños hasta los 5 años y mujeres en edad fértil de 10 a 49 años) y las Encuestas Nacionales de Factores de riesgo 2005 y 2010 (se evaluó la población adulta de 18 a 64 años. En Santa $\mathrm{Fe}$, se inició una encuesta antropómetrica en 2010, pero sólo se publicaron resultados parciales relacionados con la prevalencia de sobrepeso y obesidad (no se evaluaron aspectos vinculados a la alimentación). 
la omisión del desayuno es una de las conductas alimentarias de niños y adolescentes que se relaciona con riesgo de sobrepeso y obesidad. Otro factor condicionante en la alimentación de los niños son las cantinas escolares. En 2006, un estudio realizado en cuatro provincias argentinas en escolares de $1^{\circ}$ a $6^{\circ}$ grado encontró que un $84 \%$ de los niños consumía alimentos en la escuela, principalmente comprados en el quiosco (Oyarsun, 2010). Estudios hechos en Brasil (Fernandes, 2009), Chile (Bustos, 2010) y Argentina (Torresani, 2007) señalan que los escolares, a la hora de elegir alimentos en la escuela, prefieren aquellos que son de alta densidad de energía, grasas, sal y azúcares, y pobres en nutrientes, tales como productos envasados dulces y salados, jugos artificiales, bebidas gaseosas y helados, que son los que más abundan en los quioscos escolares. A su vez, la industria alimenticia ha multiplicado la oferta de golosinas, productos de copetín, jugos artificiales y otros alimentos que, si bien resultan muy atractivos para los niños, su ingesta excesiva termina perjudicando su salud.

Atendiendo a esta situación, en marzo de 2012 se inició un Proyecto de Extensión de Interés Social (PEIS) en la UNL denominado "Promoción de desayuno y colación saludables en escolares de la ciudad de Santa Fe", que contó con la participación de la Federación de Cooperadoras Escolares del Departamento La Capital y de dos escuelas primarias públicas de la zona céntrica de la ciudad de Santa Fe.

El equipo responsable del diseño, ejecución y evaluación del PEIS fue interdisciplinario (participaron especialistas en ciencias biológicas, en nutrición, en ciencia y tecnología de los alimentos y en estadística) y multisectorial (se realizaron vínculos con la Federación de Cooperadoras Escolares y a través de ella con las escuelas que participaron en el proyecto, y también con el Concejo Municipal de la ciudad de Santa Fe). Este trabajo interdisciplinario contribuyó a un correcto diagnóstico de situación y generó numerosas y diversas propuestas de resolución del problema planteado. La finalidad del proyecto fue contribuir a mejorar la salud y la nutrición de los niños en edad escolar, promocionar los beneficios de una alimentación saludable y resaltar la importancia de realizar un desayuno y colación de buena calidad. Teniendo como premisa que la adquisición de hábitos alimentarios saludables permitirá mejorar la calidad de vida de la comunidad escolar y prevenir la malnutrición de los niños, particularmente el sobrepeso y la obesidad, se realizó educación alimentaria nutricional. Al mismo tiempo se intentó limitar el expendio sin control de alimentos hipercalóricos para que los niños tengan la posibilidad de adquirir alimentos nutritivos que contribuyan a una alimentación saludable en el ámbito escolar.

Cabe destacar que mediante la ejecución de este proyecto se pretendía promover la aplicación de lo establecido en la Ley $N^{\circ} 26396$, de Obesidad y Trastornos Alimenticios, que en su artículo 9 especifica: "los kioscos y demás establecimientos de expendio de alimentos dentro de los establecimientos escolares deberán ofrecer productos que integren una alimentación saludable y variada, debiendo estar los mismos debidamente exhibidos".

Ley que no ha sido aplicada en todas las provincias. A su vez, el proyecto pretendía específicamente dar impulso en nuestra provincia de Santa Fe al Proyecto de Ley para promover la alimentación saludable en las escuelas de la provincia, que tuvo en dos oportunidades media sanción en la Cámara de Diputados pero que nunca alcanzó estado parlamentario.

\section{Educación alimentaria como herramienta para modificar hábitos alimentarios}

La Organización de las Naciones Unidas para la Agricultura y la Alimentación (FAO) considera que la educación es un requisito indispensable para construir un mundo dotado de seguridad alimentaria, reducir la pobreza y conservar y aumentar los recursos naturales.

La escuela ha sido considerada siempre como un sector estratégico para mejorar las conductas relacionadas con la salud. Es un ámbito propicio desde el cual se pueden llevar a cabo acciones de prevención contra la obesidad que van desde el aprendizaje hasta la oferta de alimentos en sus quioscos y comedores. La escuela posibilita incluir a una importante cantidad de niños que concurren obligatoriamente, produce alto impacto y efecto multiplicador hacia las familias, amigos y la sociedad en general. La adquisición de hábitos y conductas saludables a temprana edad asegura al individuo un crecimiento y desarrollo óptimos y salud futura.

Numerosos estudios han demostrado que las intervenciones educativas relacionadas con la alimentación y nutrición originan cambios favorables en la alimentación de los escolares. En este contexto, la educación alimentaria nutricional (EAN) surge como una herramienta capaz de ayudar, a través del conocimiento, a mejorar la calidad de los hábitos alimentarios de la población estudiantil, promoviendo la adopción voluntaria de conductas relacionadas con la nutrición que favorecen la salud y el bienestar (Borrel, 2005 y Contento, 2007). Además de informar, intenta concientizar y aumentar la motivación intrínseca de las personas para tomar un rol activo en la mejora de su propia alimentación y la de los otros buscando integrar el área biológica con el área sociocultural. En Argentina, el Ministerio de Educación de la Nación cuenta con un proyecto específico de EAN para las escuelas de Educación General Básica/Primaria impulsado por la FAO que, lamentablemente, no ha sido incluido sistemáticamente en todas las instituciones de nuestro país. 


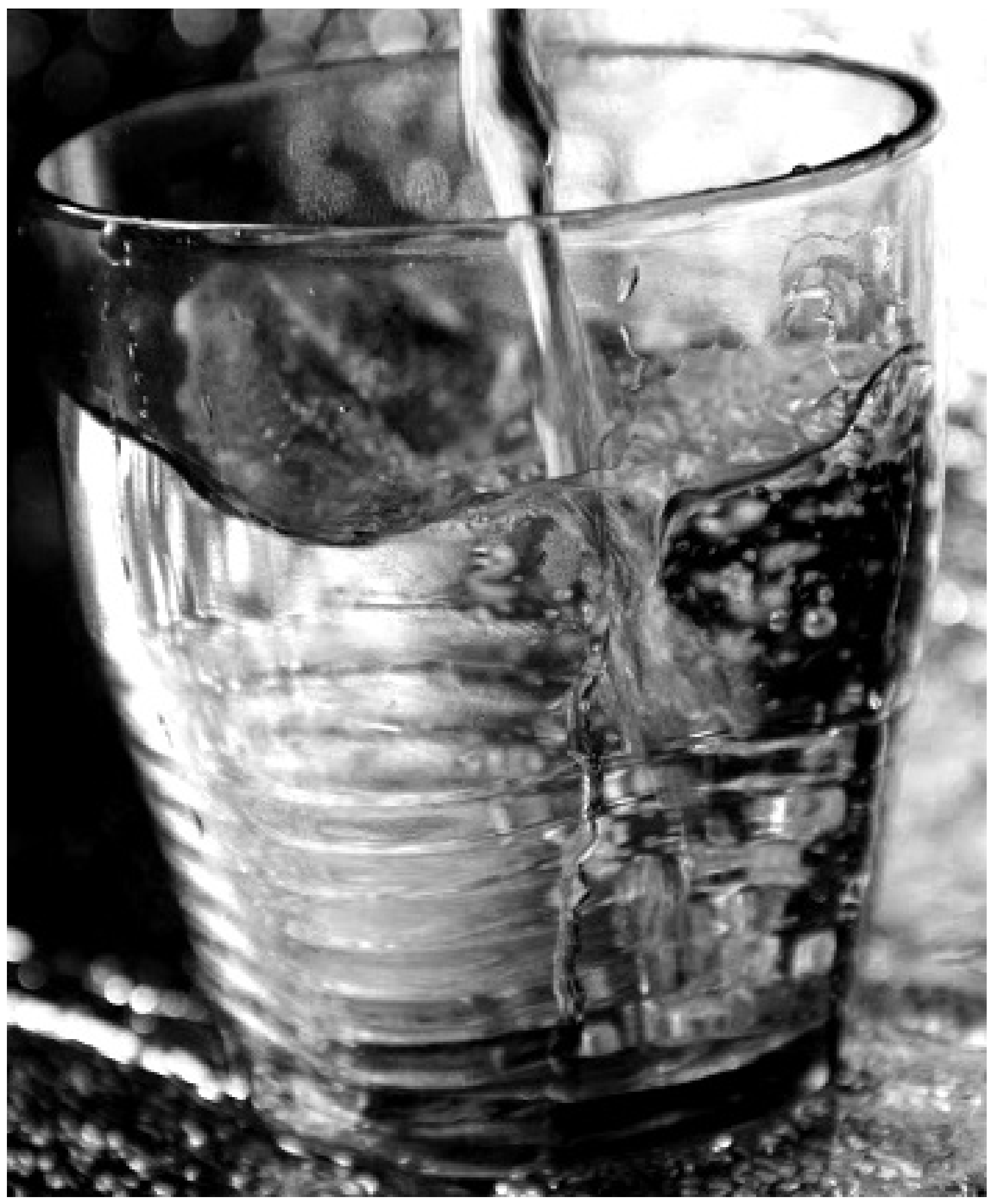




\section{Las actividades de extensión y la docencia}

Dado que uno de los pilares del proyecto era realizar educación alimentaria, consideramos que nuestra labor docente podía ser de suma utilidad no sólo para la transferencia de conocimientos a los escolares sino también para capacitar a toda la comunidad educativa para que las acciones sean sostenidas en el tiempo. Las acciones de extensión están íntimamente relacionadas con la docencia y la investigación. De hecho, el trabajo de extensión comenzó con una actividad de investigación que permitió conocer cómo desayunaban y qué alimentos compraban los niños en las escuelas con el fin de detectar las problemáticas vigentes y a partir de allí realizar los esfuerzos necesarios para introducir los cambios que se consideren pertinentes. Es imprescindible nuestra formación como docentes para realizar actividades de extensión y la interacción con los actores sociales para detectar en conjunto las problemáticas. De esa combinación surgirán nuevas ideas para proyectos de extensión e investigación.

Al inicio del PEIS se convocó a dos pasantes en extensión, cuatro tesinistas, un becario graduado y aceptaron participar 18 voluntarios del sistema de voluntariado de la UNL. Todos ellos fueron convocados a una reunión previa para comentarles los objetivos del proyecto y motivarlos en las distintas actividades a realizar. Recibieron la capacitación correspondiente, y todos los estudiantes demostraron permanentemente estar sumamente interesados en la propuesta y sugirieron numerosas nuevas actividades y formas de implementarlas. Esto enriqueció la realización del proyecto y a los estudiantes le posibilitó aplicar los conocimientos adquiridos al vincular el aprendizaje en el aula con la extensión en su formación universitaria. Aceptaron gustosamente participar en distintas actividades para dar a conocer y difundir los objetivos y resultados del proyecto. ${ }^{2}$

Todo esto nos demuestra la importancia de contar con proyectos de extensión para propiciar la formación de nuestros estudiantes universitarios. Ello permite que apliquen directamente sus saberes al entorno social y concientizarlos de la realidad del medio al cual pertenecen. Es notable el alto grado de compromiso de todos los alumnos y jóvenes graduados en cuanto a tratar de encontrar los medios para resolver problemáticas sociales tan vigentes en la actualidad.

\section{Estrategias para cumplir con los objetivos planteados} De la interacción de ideas entre los integrantes del proyecto y los estudiantes incorporados surgieron actividades adicionales a desarrollar para lograr los objetivos propuestos. La participación de los voluntarios permitió que se trabajara con grupos reducidos de alumnos en las escuelas (20-25 alumnos). Todos los integrantes participaron en la realización de encuestas para conocer cómo desayunaban los niños y qué compraban en los quioscos de las escuelas, en charlas-talleres sobre alimentación y nutrición dirigidos a los niños, padres y maestros, en entrega de colaciones saludables en numerosas oportunidades, y en la creación de juegos didácticos y su aplicación en el aula para reforzar los conocimientos en alimentación y nutrición. Adicionalmente, se orientó a los escolares para hacer videos de cocina teniendo como consigna la inclusión de frutas y verduras en las recetas.

En lo que respecta al quiosco escolar, se confeccionó una lista de alimentos recomendados para la incorporación en el quiosco saludable. La lista se logró luego de un exhaustivo relevamiento de los distintos productos que se comercializan actualmente en el mercado, incluyendo dietéticas, distribuidoras mayoristas y minoristas y panificadoras. Los productos se evaluaron en función de su calidad nutricional, del costo y de su forma de comercialización (venta individual o a granel). Se capacitó al concesionario del quiosco sobre alimentación saludable para lograr la incorporación de variedad de alimentos y la correcta ubicación de los mismos dentro de ese espacio. Se entregó material soporte que incluía una breve descripción de conceptos tales como: qué es un quiosco saludable, sus beneficios, aspectos a tener en cuenta para lograr una correcta implementación, entre otros.

Se promocionaron los nuevos productos del quiosco a través de actividades didácticas con las maestras para difundir su consumo dentro de la escuela. Se motivó a los escolares en el diseño de campañas promocionales como estrategia de venta de los productos saludables ofrecidos. Junto a los docentes, los alumnos realizaron campañas de difusión del nuevo quiosco escolar a través de afiches que contemplaban los nuevos alimentos que allí se vendían y recomendaciones nutricionales saludables.

Como parte de esta intervención se pretendió la modificación de la infraestructura del quiosco. Para poder llevarlo a cabo, se asignaron tareas a todos los grados con diferentes niveles de complejidad acordes a la edad de los niños. Se confeccionaron carteles y se creó un logo que permitió la identificación del nuevo quiosco, ahora más saludable. Se difundió la labor realizada con los alumnos en la escuela y la comunidad: la escuela organizó una jornada integradora a la que asistieron los niños de $1^{\circ}$ y $2^{\circ}$ grado con sus padres, donde se promovió un espacio más saludable y se incluyó la actividad física. Durante la jornada se elaboraron sándwiches saludables y ensaladas de frutas. Los medios de comunicación televisivos se hicieron presentes y entrevistaron a padres, directivos, docentes y concesionario del quiosco con el fin de dar a conocer la propuesta a la comunidad.
2) Tales como: el programa radial de la FM "X" de la UNL, las Jornadas de Jóvenes Investigadores, las Jornadas 40 años de la Facultad de Bioquími- ca y Ciencias Biológicas destinadas principalmente a alumnos de escuelas secundarias y público en general, en el Congreso Nacional de Nutrición. 


\section{Logros y dificultades}

Al inicio del trabajo detectamos que más de la mitad de los niños concurría a la escuela habiendo consumido un solo alimento o sin desayunar. En la escuela, la elección de la colación a media mañana no era la adecuada para completar el desayuno (Fugas, 2007). La mayoría de los niños compraba su colación en el quiosco y elegía un alimento no recomendable: sándwiches de paleta y queso, panchos, facturas, bizcochos, alfajores, golosinas (Follonier, 2013).

Luego de la intervención educativa (durante el primer año del proyecto en las dos escuelas y en el segundo año en una sola escuela) se detectaron mejoras en el nivel de conocimientos en alimentación y nutrición de los niños. Todos los alumnos aceptaron gustosamente las colaciones ofrecidas por el equipo extensionista, las que a su vez son alimentos que forman parte de un buen desayuno (frutas, leche y yogur, grisines, pan, marineras y galletitas dulces de bajo tenor graso). Sin embargo, muy pocos modificaron la calidad del desayuno en el hogar, lo cual remarca la importancia que el entorno familiar ejerce en la adquisición de hábitos alimentarios apropiados.

La implementación del quiosco saludable resultó una de las tareas con mayor dificultad durante la ejecución del presente proyecto. Ante la propuesta de cambio hubo resistencia por parte de los concesionarios de los quioscos, lo que podría estar asociado a que los intereses que se persiguen son principalmente económicos y no existe ninguna reglamentación para regular la venta de productos. A pesar de los esfuerzos durante el desarrollo de este proyecto, fueron muy pocos los alimentos sugeridos que se incorporaron definitivamente para la venta en los quioscos escolares. Por lo tanto, nos enfrentamos a dos problemas: por un lado, la oferta de alimentos que no es regulada y, por otro, la disponibilidad de dinero que tienen los niños sumada a falta de asesoramiento para realizar su compra. Es importante destacar que la mayoría de los niños en todos los niveles escolares reconoce cuáles son los alimentos considerados como no recomendables. No obstante, a la hora de comprar en el quiosco escolar los niños no aplican estos conocimientos y se dejan llevar por la sensación subjetiva del "me gusta". Hay que tener en cuenta que, si bien reconocen los alimentos no recomendables, tienen dificultad para identificar los considerados como recomendables, lo que también puede condicionar la elección al momento de comprar la colación.

\section{Políticas públicas y alimentación saludable}

Desde el comienzo del proyecto se firmó un convenio con el Concejo Municipal de la ciudad de Santa Fe para intentar trabajar en forma conjunta de modo de lograr la implementación de quioscos saludables en los establecimientos educativos. El 23 de abril de 2013, el Concejo aprobó una ordenanza con la que se procura "tutelar y proteger la salud de la población infantil y adolescente, en términos de alimentación saludable, que imparten enseñanzas de educación inicial, primaria y secundaria". Mediante esta ordenanza, el Poder Ejecutivo y el Concejo Municipal podrían instrumentar los programas y mecanismos de promoción en las escuelas y en cantinas escolares a fin de fomentar una alimentación saludable y prevenir enfermedades relacionadas con la malnutrición por exceso (sobrepeso y obesidad) y la deficiencia de macro y micro nutrientes, también denominada desnutrición oculta. En junio de 2014 se llevó a cabo una jornada que convocó a directivos de escuelas primarias de la ciudad de Santa Fe para promover una alimentación saludable dentro del ámbito escolar. En estas jornadas, nuestro grupo de extensión presentó los resultados obtenidos del trabajo y las distintas estrategias empleadas para el logro de nuestros objetivos. Otras escuelas también presentaron resultados de sus iniciativas propias para fomentar una alimentación saludable en la comunidad escolar. A raíz de ello, se realizarán acciones en conjunto para la implementación de quioscos saludables, abordando la temática desde las distintas disciplinas (tecnología, ciencias naturales, inglés, etc.). La Municipalidad, desde sus áreas de Educación y Salud, estará a disposición de las escuelas para capacitación y charlas informativas.

En tanto, el presidente de la Federación de Cooperadoras Escolares permitió la vinculación con el Ministerio de Educación de la Provincia de Santa Fe, específicamente con el secretario de Innovación Educativa y Relaciones Interinstitucionales. Pero hasta el momento no se logró un compromiso concreto para ejecutar acciones de educación alimentaria e implementación de quioscos saludables. Recientemente, el Concejo Municipal inició una serie de charlas con el Ministerio de Educación de la Provincia, por lo que se prevé que las acciones que se desarrollen en la ciudad luego puedan extenderse.

El 13 de diciembre de 2012 se sancionó la Ley № 13295 que crea el Programa Provincial Integral de Prevención, Control y Asistencia de los Trastornos Alimentarios, que en su artículo 8 establece la importancia de vincularse con el Ministerio de Educación para que, entre otros aspectos, se pueda incorporar la educación alimentaria nutricional al sistema educativo en todos los niveles, para la promoción de la actividad física a fin de evitar las conductas sedentarias de los alumnos, para el control sobre los quioscos, bufetes y demás medios de expendio de alimentos que se encuentren en los establecimientos escolares. Pero hasta el momento no ha sido implementada, en las escuelas se desconoce la existencia de esta ley, y no está claro qué organismos son los responsables de su implementación y control en las escuelas. Por otra parte, el presidente de la Federación de Cooperadoras Escolares estableció contacto con una diputada de la Comisión de Educación de la Cámara de Diputados, quien presentó en abril de 2013 el Proyecto de Ley vinculado con esta temática, retomando el anterior proyecto que fue aprobado dos veces en la Cámara de 


\section{6}

la educación alimentaria nutricional intenta concientizar y aumentar

la motivación intrínseca de las personas para tomar un rol activo en la mejora de su propia alimentación
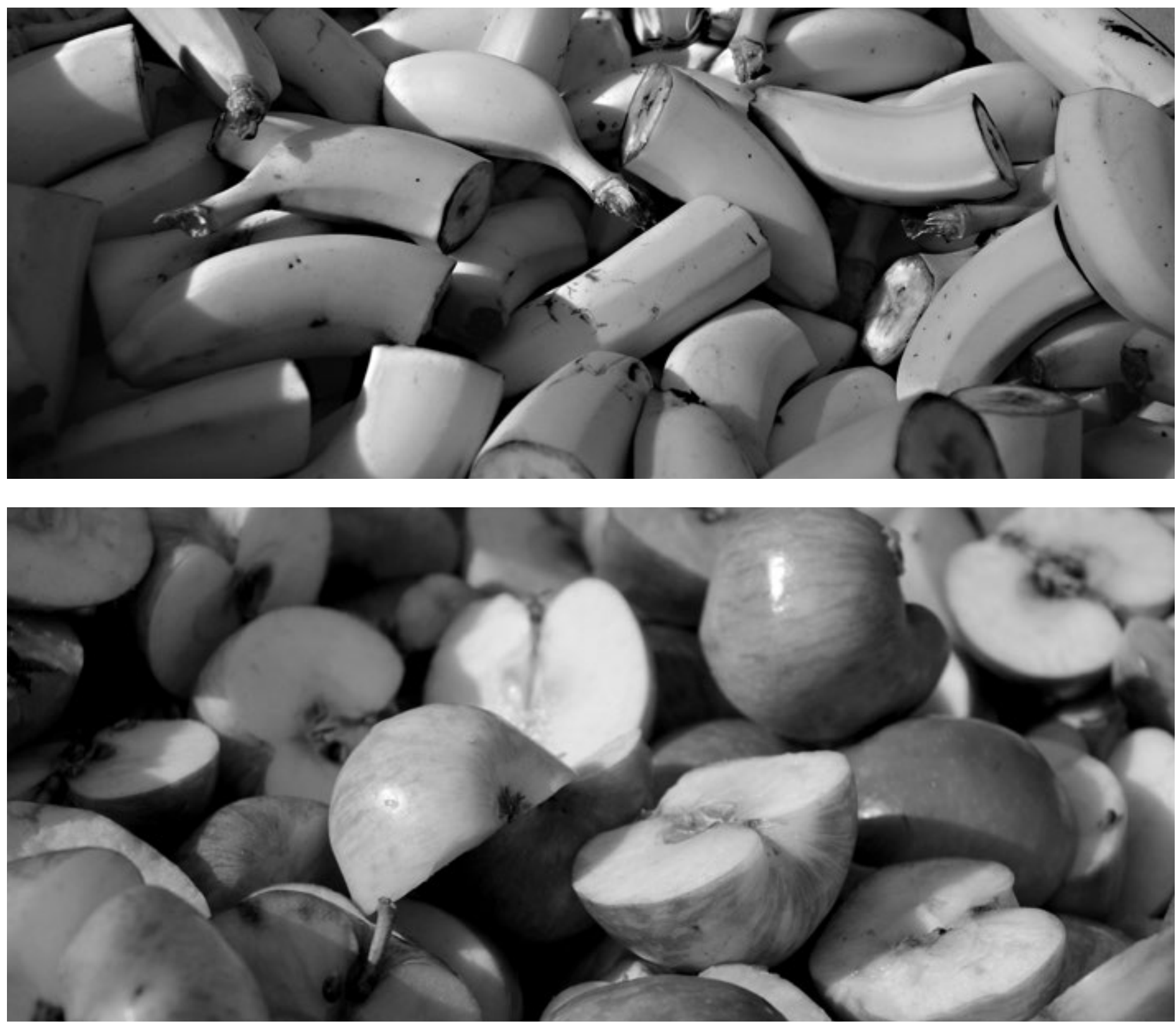


\section{6}

desde el comienzo del proyecto se firmó un convenio con el Concejo Municipal para trabajar en la implementación de quioscos saludables en los establecimientos educativos

Diputados pero nunca alcanzó estado parlamentario. Pensamos que ésta sería la forma más viable de dar solución al problema, ya que en numerosas provincias de Argentina ya tienen sancionadas e implementadas sus leyes de educación alimentaria nutricional, respecto de las cuales los Ministerios de Educación y Salud son los responsables de su implementación y del control de su cumplimiento.

\section{Perspectivas}

El cambio en los hábitos alimentarios es el fin último de la educación alimentaria nutricional. No obstante, en la mayor parte de los casos este resultado sólo se puede observar a largo plazo y en ciertas circunstancias hay que esperar incluso años para que se produzcan cambios en la población intervenida. Por ello es indispensable la implementación de políticas capaces de crear y desarrollar programas de promoción de la salud y de incorporar temáticas sobre alimentación y nutrición en la currícula escolar. Desde los equipos de extensión se pueden hacer numerosas intervenciones teóricas y prácticas para intentar establecer hábitos de vida saludable en los niños. Pero para que las intervenciones que tienen como objetivo modificar conductas alimentarias resulten efectivas es necesario abarcar la participación activa de todos los actores involucrados (niños, padres, docentes, directivos, vendedores de alimentos en las cantinas escolares, etc.). Consideramos que en este estudio los niños y jóvenes fueron los principales actores de la intervención educativa y que fue limitada la participación de padres y docentes en la mayoría de los casos. Adicionalmente, no se ha podido establecer un control más estricto en lo que respecta al expendio de alimentos en las escuelas. Por lo que se considera de vital importancia la sanción e implementación de las leyes tendientes a mejorar la alimentación en la población infantil. Lo que resulta muy gratificante es el gran interés que tiene el presidente de la Federación de Cooperadoras Escolares puesto que realmente se preocupa y ocupa de la alimentación infantil. Gracias a su interés y dedicación ha sido posible llegar a autoridades provinciales para elevar nuestra preocupación sobre la alimentación infantil y es nuestro deseo que esto ayude a obtener soluciones para esta cuestión que, en definitiva, afecta a toda la sociedad. Como intento de comenzar a solucionar el problema de los quioscos escolares, el presidente de la Federación propone que en el contrato de concesión que se firma con el encargado del quiosco escolar se agregue un inciso en donde se establezca qué alimentos deben incorporarse para su venta, siguiendo el protocolo que tiene implementado la provincia de San Luis y con asesoramiento del Colegio de Nutricionistas de Santa Fe. Además, el presidente de la Federación continúa convocando a directivos de las escuelas de la ciudad y mantiene reuniones con el Concejo Municipal para dar a conocer los resultados de este proyecto e instalar la idea de que es necesario operar cambios en todos los establecimientos escolares, tratando de que cada institución asuma un rol principal en la promoción de buenos hábitos alimentarios en su comunidad educativa.

Creemos que es absolutamente relevante establecer vínculos con el Ministerio de Salud de la provincia dados los preocupantes resultados obtenidos respecto del estado nutricional de los escolares. Se ha detectado una elevada prevalencia de sobrepeso y obesidad, situación que también se ve reflejada en la población adulta argentina. Es indispensable que se tomen medidas en etapas tempranas de la vida, ya que los hábitos alimentarios y los estilos de vida que se adquieren en la infancia tienden a perdurar durante toda la vida.

Para que las acciones sean sostenidas en el tiempo, consideramos de real importancia que se sancione la ley provincial que contempla la educación alimentaria nutricional en todos los niveles escolares y la implementación de quioscos saludables. La Universidad, a través de sus proyectos, puede plantear acciones futuras en conjunto con los Ministerios de Educación y de Salud de la provincia de Santa Fe para acompañar y apoyar la ley (con capacitación a maestros y concesionarios de quiosco, mediante el trabajo en conjunto con toda la comunidad educativa en charlas y talleres, con la interacción directa con los niños). La UNL, por medio de sus docentes y alumnos, que tienen especial interés para el trabajo social, puede acompañar y colaborar con los organismos gubernamentales en la puesta en práctica de determinadas medidas que en esta época resultan vitales para mantener la salud de la población y evitar enfermedades a futuro. 


\section{Bibliografía}

Borrel Bentz, M. R. (comp.) (2005). "Municipios Saludables. Portafolio Educativo". OPS/Ministerio de Salud y Ambiente de la Nación. Buenos Aires.

Bustos, Z. N.; Kain, B. J.; Leyton, D. B.; Olivares, C. S. y Del Río, R. F. (2010).

"Colaciones habitualmente consumidas por niños de escuelas municipalizadas: motivaciones para su elección". En Revista Chilena de Nutrición, 37(2), pp. 178-183. DOI: 10.4067/S0717-75182010000200006.

Contento, I. (2007). Nutrition Education: linking research, theory, and practice. Subdury, MA: Jones and Bartlett Publisher.

Fernandes, P. S. y otros (2009). "Evaluating the effect of nutritional education on the prevalence of overweight/obesity and on foods eaten at primary schools". In Jornal de pediatría, 85, pp. 315-321. DOI:10.2223/JPED.1917.

Follonier, M.; Bonelli, E.; Walz, F.; Martinelli, M. (2013). "Consumo de alimentos en los kioscos de escuelas primarias públicas de la ciudad de Santa Fe". En FABICIB: Revista de la Facultad de Bioquímica y Ciencias Biológicas de la UNL, 17, pp. 103-112.

Fugas, V. A.; Berta, E. E.; Walz, F.; Fortino, M. A.; Martinelli, M. I. (2013). "Hábito y calidad del desayuno en alumnos de dos escuelas primarias públicas de la ciudad de Santa Fe". En Archivos Argentinos de Pediatría, 111(6), pp. 502-507. DOI: 10.5546/aap.2013.502.

Honorable Concejo Municipal de la ciudad de Santa Fe (2013). Ordenanza

11989. Disponible en: http://www.concejosantafe.gov.ar/Legislacion/ordenanzas/ ORDE_11989.pdf

Kovalskys, I.; Indart Rougiera, P.; Amigo, M. y col. (2013). "Ingesta alimentaria y evaluación antropométrica en niños escolares de Buenos Aires". En Archivos Argentinos de Pediatría, 111(1), pp. 9-15. DOI: 10.5546/aap.2013.9.
Ministerio de Educación de Argentina. Proyecto de Educación Alimentaria y Nutricional (PAC/FAO). Proyecto Tcp/Arg/3101 (T) Educación Alimentaria y Nutricional en las Escuelas de Educación General Básica/Primaria.

Ministerio de Salud de la Nación (2008). Ley de Obesidad y Trastornos Alimenticios. Disponible en: http://www.msal.gov.ar/argentina-saludable/pdf/leyobesidad.pdf

Ministerio de Salud de la Nación (2011). Encuesta Nacional de Factores de Riesgo. Disponible en: http://www.msal.gov.ar/fesp/descargas_home/seg_encuesta_nac_ factores_riesgo_2011.pdf

Ministerio de Salud de la Nación. Argentina saludable. Disponible en: http://www. msal.gov.ar/argentina-saludable/

Organización Mundial de la Salud (2006). "Obesity and overweight". Fact sheet NN 311. Disponible en: http://www.who.int/mediacentre/factsheets/fs311/en/ Organización Mundial de la Salud/Organización para la Alimentación y la Agricultura (2003). "Dieta, nutrición y prevención de enfermedades crónicas". Serie de Informes Técnicos 916. Ginebra.

Oyarzun, M. T.; Yofre, F. (2010). "Aprender a alimentarse". En Alimentos Argentinos 47, pp. 9-12.

Proyecto de Ley Educación Alimentaria Nutricional. Provincia de Santa Fe. Disponible en: http://www.veronicabenas.com.ar/Educacion\%20Alimentaria.pdf Szer, G., Kovalskys, I. y De Gregorio M. (2010). "Prevalencia de sobrepeso, obesidad y su relación con hipertensión arterial y centralización del tejido adiposo en escolares". En Archivos Argentinos de Pediatría, 108(6), pp. 492-498.

Torresani M. E. et al. (2007). "Consumo en cadena de comidas rápidas y kioscos: preferencias de escolares y adolescentes de nueve colegios privados de Capital Federal y Gran Buenos Aires". En Archivos Argentinos de Pediatría, 105(2), pp. 109-114. 